\section{Finish restorations faster}

In modern dentistry, time is always of the essence, so why not choose a restorative material that streamlines the way you work and provides a number of excellent benefits?

3M Ketac Universal Aplicap Glass Ionomer Restorative is an efficient choice.

The material has been specifically designed for single placement use, reducing the number of steps so that it's a great option when treating patients of all ages. Its unique low stickiness means that it adheres to the cavity but not dental instruments, aiding accurate placement. Plus, as it is self-cure, it helps to ensure fast, effective treatment.

Another benefit of 3M Ketac Universal Aplicap Glass Ionomer Restorative is that it is incredibly versatile and offers lasting protection. Not only can it be used in both Class I and Class II restorations, but it releases fluoride for up to two years after placement for extra defence against decay.

For more information, call 08705360036 or visit https://www.3m.co.uk/3M/en_GB/ dental-uk/products/glass-ionomers/ketacuniversal/. $3 \mathrm{M}$ and Ketac are trademarks of the $3 \mathrm{M}$ Company.

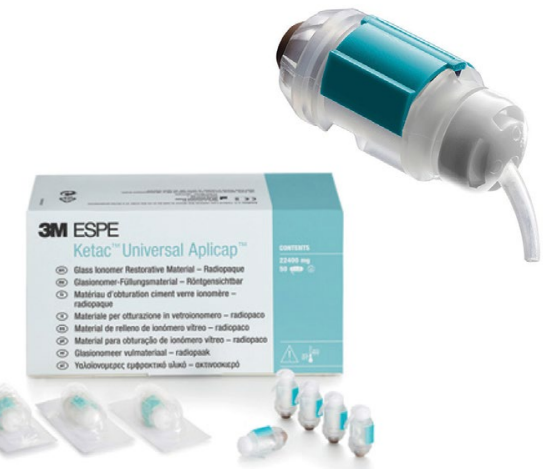

\title{
New implant gives immediate confidence
}

This January, Dentsply Sirona is delighted to launch its brand new DS PrimeTaper Implant System to the UK and Irish markets.

DS PrimeTaper is a premium tapered implant system with a progressive thread design that enables it to cut and engage with the bone to support immediate loading and shorter procedure times.

Michael Norton BDS FCS RCS(Ed) said: 'The DS PrimeTaper implant draws beautifully into the osteotomy providing progressive build-up in torque up to the $35-45 \mathrm{~N}-\mathrm{cm}$ range that is high, but still kind to the bone. It surprised me just how smooth it was, even in compromised bone.

Building on the many highly praised features of Dentsply Sirona's existing implant range - including the predictable OsseoSpeed surface which delivers outstanding marginal bone maintenance - the advanced tapered, progressive thread design delivers cutting, condensing and conserving functions for ultimate osseointegration success.

Simple drill protocols and a unique interface design that provides three different options for abutment placement and indexing makes working with DS PrimeTaper straightforward and timesaving. This enables clinicians to offer their patients fast, efficient and aesthetic immediate load options for both single and multiple implant cases with absolute confidence.

Such a winning combination will make DS PrimeTaper a force to be reckoned with in the premium implant dentistry market this year and beyond.

To find out more about Dentsply Sirona's advanced DS PrimeTaper Implant System, visit www. dentsplysirona.com/dsprimetaper.

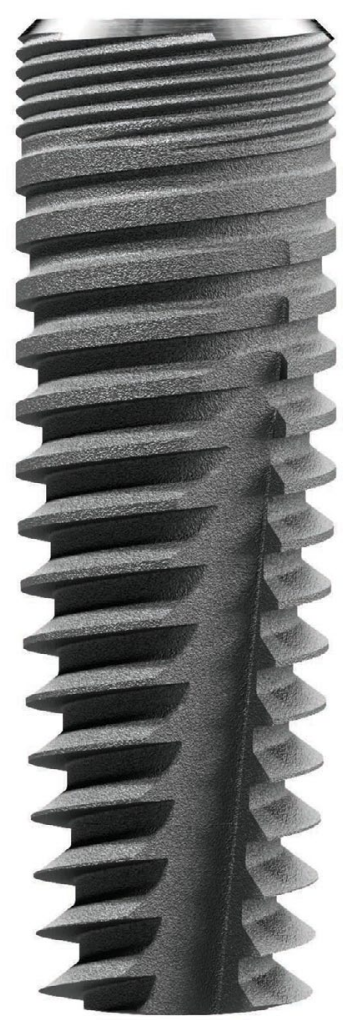

\section{Enhanced aesthetic restorations}

Tokuyama are the only composite manufacturer to use patented spherical filler particles within their composite materials, each variant utilising spherical particles of different diameters to maximise their optical and physical properties for the desired indication.

In addition to optimised optical properties resulting in enhanced aesthetic restorations, Tokuyama's spherical filler particles offer other significant advantages compared with the irregular-shaped filler particles used by all other manufacturers. They are quicker and easier to pack into nooks and crannies, reducing the risk of voids; easier to sculpt and carve, producing enhanced morphology of the final restoration; and a much smoother surface finish which has a natural high sheen that requires minimal if any polishing.

Tokuyama's development of spherical filler particles has culminated in Omnichroma, the world's only colourless universal composite. It is the only universal composite that can match every tooth colour no matter what shade of white. In addition to all the inherent advantages associated with Tokuyama's patented spherical filler particles, employing Omnichroma within your practice means freedom from time-consuming and subjective shade-matching procedures; money saved on overheads and reduced waste from expired rarely used Vita shades; and no need to replace restorations after tooth bleaching.

For more information about the complete Tokuyama range, including Omnichroma, contact your local Trycare Representative, call 01274885544 or visit www.trycare.co.uk. 